\title{
Metabolic engineering of the purine biosynthetic pathway in Corynebacterium glutamicum results in increased intracellular pool sizes of IMP and hypoxanthine
}

\author{
Susanne Peifer ${ }^{1}$, Tobias Barduhn ${ }^{1}$, Sarah Zimmet ${ }^{1}$, Dietrich A Volmer ${ }^{2}$, Elmar Heinzle ${ }^{1}$ and Konstantin Schneider ${ }^{\text {1* }}$
}

\begin{abstract}
Background: Purine nucleotides exhibit various functions in cellular metabolism. Besides serving as building blocks for nucleic acid synthesis, they participate in signaling pathways and energy metabolism. Further, IMP and GMP represent industrially relevant biotechnological products used as flavor enhancing additives in food industry. Therefore, this work aimed towards the accumulation of IMP applying targeted genetic engineering of Corynebacterium glutamicum.
\end{abstract}

Results: Blocking of the degrading reactions towards AMP and GMP lead to a 45-fold increased intracellular IMP pool of $22 \mu \mathrm{mol} \mathrm{g}_{\mathrm{CDW}}^{-1}$. Deletion of the pgi gene encoding glucose 6-phosphate isomerase in combination with the deactivated AMP and GMP generating reactions, however, resulted in significantly decreased IMP pools $\left(13 \mu \mathrm{mol} \mathrm{g}_{\mathrm{CD}}^{-1}\right)$. Targeted metabolite profiling of the purine biosynthetic pathway further revealed a metabolite shift towards the formation of the corresponding nucleobase hypoxanthine $\left(102 \mu \mathrm{mol} \mathrm{g}_{\mathrm{CDW}}^{-1}\right)$ derived from IMP degradation.

Conclusions: The purine biosynthetic pathway is strongly interconnected with various parts of the central metabolism and therefore tightly controlled. However, deleting degrading reactions from IMP to AMP and GMP significantly increased intracellular IMP levels. Due to the complexity of this pathway further degradation from IMP to the corresponding nucleobase drastically increased suggesting additional targets for future strain optimization.

Keywords: Purine accumulation, Metabolic engineering, Corynebacterium glutamicum, Targeted metabolomics, Metabolic flux analysis

\section{Background}

The diverse class of purine intermediates comprises phosphorylated nucleotides, nucleosides and nucleobases, exhibiting multiple functions in the cellular system [1]: for example, they serve as transmitters of the genetic information [2], as phosphate group donors, are involved in signal mediation [3] and ensure the energy supply of the living cell [4]. Thus, these intermediates are involved in almost every aspect of cellular metabolism. Furthermore, some of these compounds possess

\footnotetext{
* Correspondence: ko.schneider@mx.uni-saarland.de

${ }^{1}$ Biochemical Engineering Institute, Saarland University, Campus A1.5, 66123, Saarbrücken, Germany

Full list of author information is available at the end of the article
}

secondary functions as flavor enhancing substances, i.e. IMP and GMP [5-7], and act as potential drugs in medical therapy, e.g. inosine and purine analogs [8-10]. Due to their immense pharmaceutical and biotechnological potential, these compounds have been successfully synthesized over the past decades via fermentative production employing Corynebacteria, especially Corynebacterium ammoniagenes [11-13].

Their de novo synthesis, however, is energetically expensive and imbalances in their pool sizes are reported to be directly linked to increased mutation rates [14], which eventually leads to constant pool sizes [2]. These are maintained by a balanced system with two compensatory operating parts: the de novo biosynthesis pathway,

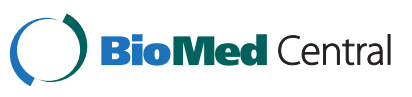


generating the purine intermediates from central carbon metabolism, and the salvage pathway, which can replenish corresponding pools (Figure 1) [6,7,10,15].

De novo nucleotide synthesis begins with the activation of the pentose phosphate pathway (PPP) intermediate and direct precursor ribose 5-phosphate [16], which is further converted to IMP in a twelve-step biosynthetic process with concomitant consumption of $10 \mathrm{~mol}$ ATP. AMP and GMP are derived from IMP as depicted in Figure 1 [1,17]. The salvage pathway, however, converts extracellular nucleobases or degraded purine compounds into the corresponding nucleosides and nucleotides $[11,18]$, thereby preventing energetically expensive de novo synthesis of these compounds.

Since primary metabolism is designed and optimized to deliver and sustain a more or less consistent distribution of metabolites (described as network rigidity [19]) which can be used as cellular building blocks, redox equivalents, energy and co-factors - any kind of overproduction or accumulation basically targets the deregulation of the underlying metabolism. In the present study, a strategy for increased IMP accumulation was developed by focusing on modifying the general key factors for overproduction: (i) increasing the precursor availability; (ii) de-bottlenecking regulation-restricted branch points, and (iii) deactivating the depleting reactions. The goal of this study was to identify essential nodes of this pathway and to uncover impacts from central and purine metabolism.
Applying genetic engineering and in-depth metabolome analysis of the purine pathway intermediates, information of the extremely well-regulated purine pathway should be gathered $[1,17,20,21]$. However, the underlying mechanisms and the contributions of different branches are not well described yet. In addition, identification of individual regulation factors [22,23] operating at different hierarchic levels [24] is challenging due to the comprehensive connectivity of the metabolic network. The interconnectivity of metabolome and fluxome therefore provides valuable insights into global dynamics $[25,26]$ resulting from single and multiple perturbations in the central and branch metabolism [27].

\section{Results}

Metabolic snapshots of the purine pathway in wild type

\section{C. glutamicum}

Intracellular metabolite concentrations in C. glutamicum were quantified after whole culture extraction using LCESI-MS/MS [28]. In order to verify balanced growth conditions $[29,30]$ and stable intracellular metabolite concentrations [31,32], respectively, quantification was performed for two different biomass concentrations, resulting in four biological replicates for each biomass concentration. The corresponding concentrations of nucleotides, nucleosides and nucleobases related to the cell dry weight are listed in Table 1.

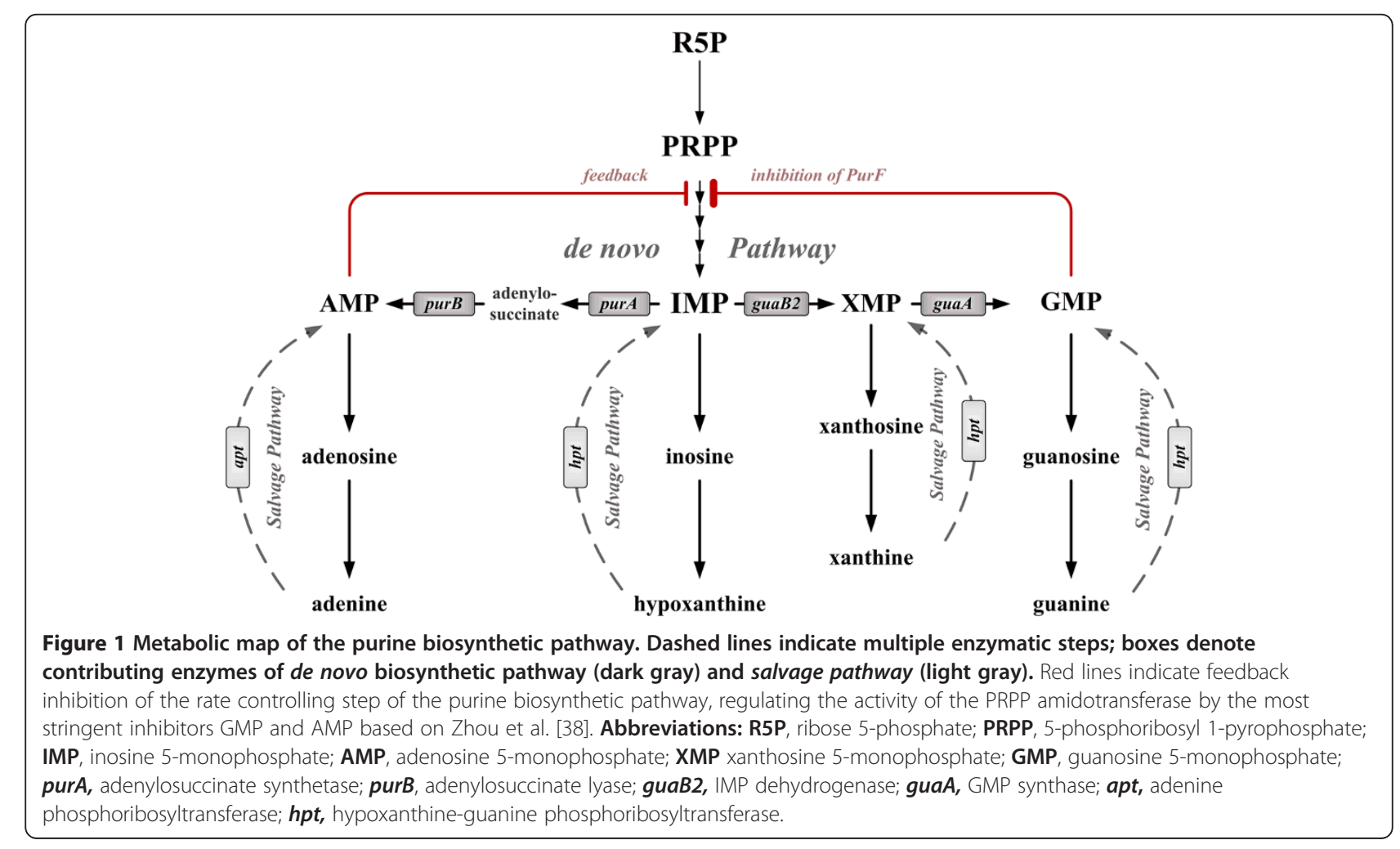


Table 1 Concentrations of purine intermediates [ $\mu \mathrm{mol} \mathrm{g}_{\mathrm{CDw}}^{-1}$ ] determined for C. glutamicum wild type (ATCC 13032) and site-directed mutants ${ }^{a}$

\begin{tabular}{|c|c|c|c|c|c|c|c|}
\hline \multirow[t]{2}{*}{ Compound } & \multicolumn{7}{|c|}{ Concentration $\left[\mu \mathrm{mol} \mathrm{g}_{\mathrm{CDW}}^{-1}\right]$} \\
\hline & ATCC 13032 & $\Delta p g i$ & purF $^{K 348 Q}$ & $\Delta p u r A^{c}$ & $\triangle g_{u a B 2}{ }^{c}$ & $\triangle p u r A \Delta g u a B 2$ & $\begin{array}{l}\triangle p u r A \Delta g u a B 2 \\
p_{u r F}^{K 348 Q}{ }_{\Delta p g i}\end{array}$ \\
\hline AMP & $8.13 \pm 0.50$ & $9.07 \pm 0.52$ & $10.24 \pm 0.78$ & $10.66(17.93) \pm 0.22(1.18)$ & $10.11(27.79) \pm 0.05(2.03)$ & $3.69 \pm 0.54$ & $3.57 \pm 0.20$ \\
\hline adenosine & $0.10 \pm 0.01$ & $0.09 \pm 0.01$ & $0.12 \pm 0.02$ & $0.13 \pm 0.01$ & $0.21 \pm 0.01$ & $0.05 \pm 0.00$ & $0.15 \pm 0.03$ \\
\hline adenine & $2.63 \pm 0.25$ & $3.04 \pm 0.11$ & $3.36 \pm 0.30$ & $3.04(5.58) \pm 0.38(0.26)$ & $4.03(8.32) \pm 0.13(0.53)$ & b & b \\
\hline GMP & $2.63 \pm 0.16$ & $3.65 \pm 0.17$ & $4.75 \pm 0.27$ & $9.47(21.10) \pm 0.12(2.03)$ & $3.92(2.33) \pm 0.19(0.24)$ & $0.65 \pm 0.07$ & $0.51 \pm 0.03$ \\
\hline guanosine & $0.04 \pm 0.00$ & b & $0.03 \pm 0.00$ & $0.03 \pm 0.01$ & $0.06 \pm 0.01$ & $0.10 \pm 0.01$ & $0.23 \pm 0.02$ \\
\hline guanine & $3.27 \pm 0.28$ & $3.85 \pm 0.17$ & $5.08 \pm 0.61$ & $5.07(10.27) \pm 1.45(0.62)$ & $35.67(9.38) \pm 1.65(0.64)$ & b & b \\
\hline IMP & $0.49 \pm 0.06$ & $0.42 \pm 0.03$ & $0.55 \pm 0.05$ & $30.72(43.99) \pm 0.48(3.32)$ & $6.39(37.16) \pm 0.21(3.48)$ & $21.91 \pm 0.89$ & $12.88 \pm 0.74$ \\
\hline inosine & $0.13 \pm 0.05$ & $0.21 \pm 0.04$ & $0.38 \pm 0.12$ & $0.28 \pm 0.06$ & $0.85 \pm 0.06$ & $1.26 \pm 0.08$ & $2.53 \pm 0.19$ \\
\hline hypoxanthine & $0.28 \pm 0.03$ & $0.29 \pm 0.03$ & $0.28 \pm 0.03$ & $11.87(17.92) \pm 1.00(1.26)$ & $3.55(16.19) \pm 0.04(1.34)$ & $80.65 \pm 3.19$ & $101.53 \pm 1.09$ \\
\hline XMP & b & b & $b$ & b & n. d. & n. d. & n. d. \\
\hline xanthosine & b & $b$ & b & b & n. d. & n. d. & n. d. \\
\hline xanthine & $0.12 \pm 0.01$ & $0.22 \pm 0.01$ & $0.11 \pm 0.00$ & $0.17 \pm 0.00$ & n. d. & n. d. & n. d. \\
\hline
\end{tabular}

${ }^{a}$ Concentrations and corresponding standard deviations were determined using 4 biological replicates for each compound and standardized to the cell dry weight.

${ }^{b}$ Below detection limit.

c Purine intermediate concentrations determined for C. glutamicum $\triangle$ purA and C. glutamicum $\triangle$ guaB2 represent maximum values obtained for two biomass concentrations for each strain: numbers on top correspond to the first biomass concentration ( $1.3 \mathrm{~g} \mathrm{~L}^{-1}$ for $C$. glutamicum $\triangle$ purA and $1.1 \mathrm{~g} \mathrm{~L}^{-1}$ for $C$. glutamicum $\triangle$ guaB2) and numbers in brackets for second biomass concentration (1.9 $\mathrm{g} \mathrm{L}^{-1}$ for C. glutamicum $\triangle$ purA and $1.8 \mathrm{~g} \mathrm{~L}^{-1}$ for $C$. glutamicum $\triangle$ guaB2).

n. d. not detected.

All compounds except xanthosine and XMP, which were below the detection limit, were quantified with a precision better than 12\%. AMP and GMP, as central building constituents for DNA and RNA and co-substrates for various enzyme reactions $[2,3]$, showed the highest concentration ranges of 8.13 and $2.63 \mu \mathrm{mol} \mathrm{g}_{\mathrm{CDW}}^{-1}$, respectively. Other intermediates varied within a broader concentration range from 0.04 to $3.27 \mu \mathrm{mol} \mathrm{g}_{\mathrm{CDW}}^{-1}$. The lowest concentrations were detected for nucleosides reaching up to $0.04 \mu \mathrm{mol} \mathrm{g} \mathrm{g}_{\mathrm{CDW}}^{-1}$ for guanosine.

Several genetic perturbations aiming at various metabolic key nodes were performed to evaluate the effects and impacts of changing activities of different metabolic branches of the metabolism on the intracellular purine pool sizes.

\section{Manipulating the main carbon flux distribution}

The first implemented modification affected the glucose 6-phosphate branch point and focused on amending the availability of the immediate precursor of IMP, ribose 5phosphate. The carbon flux distribution was rerouted by deleting the first enzyme of the glycolysis, the glucose 6phosphate isomerase encoded by the pgi gene, resulting in an entry block into glycolysis at that stage and thus restricting the metabolization of glucose 6-phosphate exclusively by the PPP. Growth parameters are depicted in Table 2. The specific growth rate of C. glutamicum $\Delta p g i$ was slightly reduced compared to the wild type strain, as was biomass formation.
Phenotypic analysis was further extended towards the determination of free intracellular purine pool sizes and a metabolic profile based on these analyses was generated (Table 1). Intracellular concentrations related to cell dry weight showed only minor changes compared to wild type C. glutamicum. GMP and AMP concentrations were slightly increased in C. glutamicum $\Delta p g i$, as were amounts of adenine, guanine and inosine. Adenosine and guanosine showed decreased concentrations. IMP and hypoxanthine were not affected significantly.

\section{Targeted perturbations of the purine biosynthetic pathway}

Further genetic manipulations aimed at the purine pathway itself, to estimate immediate effects emanating from this metabolic branch. The first target was the purF gene encoding the PRPP amidotransferase. This enzyme is generally regarded to act as key controller in the purine de novo synthesis, regulated by a feedback inhibition mainly caused by GMP and AMP [7,33]. An experimentally identified amino acid substitution (K326Q) in the purF gene is reported to offset this feedback inhibition by AMP and GMP in E. coli [34] and was incorporated into the corresponding position in the purF gene of $C$. glutamicum. In order to check for evolutionary conserved coding regions, a sequence alignment covering the complete amino acid sequence was performed [34]. Therefore, six organisms exhibiting a broad phylogenetic distinction, including Escherichia coli, Corynebacterium 
Table 2 Growth parameters for C. glutamicum ATCC 13032 and site-directed mutants

\begin{tabular}{|c|c|c|c|}
\hline \multirow[t]{2}{*}{ Strain } & \multicolumn{3}{|c|}{ Parameter } \\
\hline & $\mu$ & $Y_{x / s}$ & $q_{s}$ \\
\hline ATCC 13032 & $0.443 \pm 0.004$ & $0.095 \pm 0.004$ & $4.67 \pm 0.09$ \\
\hline$\Delta p g i$ & $0.376 \pm 0.001$ & $0.090 \pm 0.002$ & $4.18 \pm 0.02$ \\
\hline purF $^{K 348 Q}$ & $0.440 \pm 0.008$ & $0.091 \pm 0.001$ & $4.82 \pm 0.06$ \\
\hline$\Delta p u r A$ & $0.455 \pm 0.006$ & $0.090 \pm 0.001$ & $5.05 \pm 0.02$ \\
\hline$\triangle g u a B 2$ & $0.320 \pm 0.007$ & $0.073 \pm 0.004$ & $4.38 \pm 0.03$ \\
\hline$\triangle p u r A \quad \triangle g u a B 2$ & $0.317 \pm 0.006$ & $0.075 \pm 0.001$ & $4.24 \pm 0.01$ \\
\hline $\begin{array}{l}\Delta \text { purA } \triangle g u a B 2 \\
\text { purF }^{K 348 Q} \Delta p g i\end{array}$ & $0.146 \pm 0.003$ & $0.076 \pm 0.003$ & $1.92 \pm 0.02$ \\
\hline
\end{tabular}

Given data represent mean values obtained from two parallel cultivations with corresponding standard deviations: specific growth rate $(\mu)\left[h^{-1}\right]$; biomass yield $\left(\mathrm{Y}_{\mathrm{X} / \mathrm{S}}\right)\left[\mathrm{g} \mathrm{mmol}^{-1}\right]$ and the specific glucose consumption rate $\left(\mathrm{q}_{\mathrm{S}}\right)\left[\mathrm{mmol} \mathrm{g}^{-1} \mathrm{~h}^{-1}\right]$.

glutamicum, Corynebacterium ammoniagenes, Bacillus subtilis, Salmonella typhimurium and Saccharomyces cerevisiae, were analyzed. A conserved region comprising at least ten amino acids was identified for all organisms including the identified location of the amino acid substitution K326Q in E. coli described by Zhou et al. [34]. The corresponding substitution K348Q in C. glutamicum was inserted into the genomic DNA. Verification of the base exchange was carried out by sequencing.

The amino acid substituent C. glutamicum purFK348Q $\left(\right.$ purF $\left.{ }^{K 348 Q}\right)$ showed balanced growth with stable biomass formation and growth rate (Table 2). Intracellular purine intermediate pool sizes remained almost unchanged for adenosine, guanosine and hypoxanthine (Table 1). The most striking changes were observed for intracellular inosine concentration which increased 2.9-fold. Compared to the wild type strain, the IMP concentration, however, showed only a minor change (10\% increase). Intracellular concentrations of AMP and its corresponding nucleobase adenine increased by approximately $25 \%$ each. GMP and guanine pool sizes in C. glutamicum purF ${ }^{K 348 Q}$ increased significantly by $80 \%$ and $55 \%$, respectively, compared to the wild type, indicating a global shift towards the main intermediates GMP, AMP and inosine. Direct determination of the in vitro activity of PurF following the method of Matsui et al. [35], employing HPLC quantitation of substrate (glutamine) and product (glutamate) concentrations [36] over time in crude cell extracts, resulted in tremendous background activity, not permitting kinetic studies. In addition, heterologous expression using Escherichia coli as expression system resulted in enzyme inactivation, probably due to complex posttranslational events. Further studies will however focus on the determination of the in vitro activity.

In addition to the deregulation of PurF, further genetic manipulations aimed at IMP degradation, affecting the synthesis of IMP-derived GMP and AMP. The biosynthesis of both intermediates was thus shifted from de novo biosynthesis to the purine salvage pathway, employing the two enzymes adenosine-phosphoribosyltransferase (APRT) and hypoxanthine-guanine-phosphoribosyltransferase (HGPRT) catalyzing the synthesis of AMP and GMP/IMP via a condensing reaction of adenine and guanine/hypoxanthine with PRPP maintaining a sustained supply of the necessary precursors. These genetic manipulations, however, lead to auxotrophies for adenine and/or guanine [35,37].

As initial strategy, the single gene deletions of the adenylosuccinate synthetase (purA) (catalyzing the conversion of IMP to AMP) and of the IMP-dehydrogenase (guaB2) (catalyzing the reaction of IMP to GMP) were analyzed. Due to the generated auxotrophies, the medium had to be supplied with adenine and guanine and resulting phenotypes based on intracellular concentrations of the corresponding purine pathway intermediates were analyzed (Table 1).

The metabolic shift from de novo purine biosynthesis to the salvage pathway did not show any significant changes in the growth rate of the purA deletion strain. The guaB2 deletion strain, however, showed a drastic response after redirecting GMP formation to the salvage pathway: biomass yield was reduced by $25 \%$ $\left(0.073 \mathrm{~g} \mathrm{~mol}_{\text {glucose }}^{-1}\right)$ of the wild type yield and the specific growth rate even dropped by $30 \%$ of the wild type rate, reaching a stable rate of $0.32 \mathrm{~h}^{-1}$, thus indicating a restricted compensation efficiency of the salvage pathway concerning the GMP supply. Metabolic phenotyping revealed even more distinct alterations: most strikingly both deletion mutants were characterized by unbalanced intracellular pools sizes, exhibiting transient concentration changes which could not be related to biomass formation (Table 1). Degradation inefficiency of IMP towards the following nucleotides (GMP and AMP) led to maximal intracellular IMP concentrations of $44 \mu \mathrm{mol}$ $\mathrm{g}_{\mathrm{CDW}}^{-1}(\triangle p u r A)$ and $37 \mu \mathrm{mol} \mathrm{g}_{\mathrm{CDW}}^{-1}(\Delta g u a B 2)$ corresponding to a 90 - and 80 -fold increase compared to the wild type strain. Hypoxanthine concentrations were affected likewise.

The unbalanced metabolic state observed in C. glutamicum $\triangle$ purA and C. glutamicum $\triangle g u a B 2$, was further investigated in the double deletion mutant C. glutamicum $\triangle p u r A \triangle g u a B 2$, exhibiting auxotrophies for adenine as well as for guanine. Specific growth parameters of the double deletion mutant, i.e. biomass yield ( $\left.0.075 \mathrm{~g}_{\mathrm{CDW}} \mathrm{mmol}_{\text {glucose }}^{-1}\right)$ and specific growth rate $\left(0.317 \mathrm{~h}^{-1}\right)$, were consistent with those obtained for C. glutamicum $\triangle g u a B 2$, indicating a mutual growth-related effect. However, the metabolic fingerprint for C. glutamicum $\triangle$ purA $\triangle$ guaB2 exhibited remarkable differences (Table 1): the unbalanced metabolic behavior observed in C. glutamicum $\triangle$ purA and $C$. glutamicum $\triangle$ guaB2 was completely reestablished resulting in constant intracellular purine intermediate 
concentrations for all substances quantified. The double deletion mutant showed a decreased intracellular IMP pool of $21.9 \mu \mathrm{mol} \mathrm{g} \mathrm{gDW}_{\mathrm{CD}}^{-1}$ compared to the single gene deletion mutants which corresponds to a 44-fold increase compared to the wild type strain. The IMP reduction was accompanied by a significant increase of the inosine concentration (9.7-fold compared to the wild type strain) and especially hypoxanthine (290-fold compared to the wild type strain), indicating a metabolic shift from main purine intermediates; i.e., the nucleotides, to the corresponding degradation products (nucleobases).

\section{Extended metabolic manipulations - combination of central pathway and branch pathway modifications}

Finally, the combined influence of the genetic modifications described above was investigated. The rationally designed mutant C. glutamicum $\triangle$ purA $\triangle g u a B 2$ purF $^{K 348 Q} \Delta p g i$ is characterized by the amino acid substitution (purFK348Q), supposed to affect the regulatory key enzyme for the de novo synthesis. In addition, the mutant exhibits gene deletions for the initiating reaction of glycolysis ( $p g i)$ and for the converting reactions leading from IMP to the successive purine nucleotides AMP (purA) and GMP (guaB2).

The biomass yield of $0.076 \mathrm{~g}_{\mathrm{CDW}} \mathrm{mmol}_{\text {glucose }}^{-1}$ for C. glutamicum $\triangle p u r A \Delta g u a B 2$ purF ${ }^{K 348 Q} \Delta p g i$ was consistent with those obtained for C. glutamicum $\triangle$ purA $\triangle g u a B 2$ and C. glutamicum $\triangle g u a B 2$. However, the specific growth rate was reduced to $0.146 \mathrm{~h}^{-1}$. In contrast to the two single gene deletion mutants $(\triangle p u r A$ and $\triangle g u a B 2)$, the previously observed IMP decline observed in the double deletion mutant $C$. glutamicum $\triangle p u r A$ $\triangle g u a B 2$ was even more pronounced in the mutant containing the four genetic modifications (Table 1). In conjunction with these findings, an increased IMP degradation towards the corresponding nucleoside inosine and nucleobase hypoxanthine was observed.

\section{Quantification of intracellular carbon flux distributions}

Alterations of in vivo carbon flux distributions were investigated in order to assess the impact on the purine intermediate profile. The network contained reactions of glycolysis, TCA cycle, anaplerosis and PPP. Estimation of intracellular flux distributions was carried out based on measurable extracellular rates, i.e. glucose consumption, biomass and by-product formation as well as the mass isotopomer data obtained from ${ }^{13} \mathrm{C}$ enriched cellular protein. Initial values were statistically varied and multiple parameter estimations were performed to guarantee finding of the global minimum solution.

The metabolic carbon flux distributions determined for the strains C. glutamicum ATCC 13032 (wild type) and C. glutamicum $\triangle p$ gi, C. glutamicum $\triangle$ purA $\triangle$ guaB2 and C. glutamicum $\triangle$ purA $\triangle$ guaB2 purF ${ }^{K 348 Q} \Delta p g i$ are illustrated in Figure 2. Metabolic and isotopic steady state was validated for all strains analyzed by ${ }^{13} \mathrm{C}$ MFA. Metabolic steady state was assessed by constant biomass and product yields (Table 2), constant intracellular metabolite concentrations (Table 1) and exponential growth (Table 2). Isotopic steady state was assured comparing mass isotopomer distributions of amino acids for two biomass concentrations. Mass isotopomer fractions of derivatized alanine, glycine, valine, threonine, phenylalanine and serine - determined for C. glutamicum ATCC 13032 (wild type) - are highlighted as illustrative examples in Figure 3. Isotopic steady states for C. glutamicum $\triangle p$ gi, C. glutamicum $\triangle$ purA $\triangle$ guaB2 and C. glutamicum $\triangle p u r A \Delta g u a B 2$ purF ${ }^{K 348 Q} \Delta p g i$ were assured likewise and exhibited comparable results (data not shown).

Deletion of the pgi gene strongly affected the carbon flux distribution and caused a complete carbon redirection towards the PPP. The reflux of ribose 5phosphate via the non-oxidative part of the PPP entering glycolysis was almost tripled. Activity of the TCA cycle was only slightly affected, showing a small reduction from $47.3 \%$ in the wild type strain to $45.7 \%$ in the pgi deletion mutant.

In contrast, the double deletion mutant C. glutamicum $\triangle p u r A \triangle g u a B 2$, possessing an intact pgi gene, exhibited the lowest flux through the oxidative part of the PPP (29.3\%), which almost exclusively accounted for biomass formation. The relative reflux into glycolysis was less than $10 \%$. TCA cycle activity was increased to $88 \%$. The strain C. glutamicum $\triangle$ purA $\triangle$ guaB2 purF $^{K 348 Q} \Delta p g i$ showed a carbon flux redirection from glycolysis towards the PPP (98.0\%) caused by the pgi gene deletion.

\section{Perturbation-caused alterations of the NADH metabolism}

The inserted genetic changes also altered the redox metabolism, especially regarding the $\mathrm{NADH} / \mathrm{FADH}_{2}$ (XADH) supply. A surplus was detected in all strains investigated, which could be used for ATP generation by oxidative phosphorylation. However, the relative NADH production exhibited significant differences among the modified strains (Figure 4): C. glutamicum ATCC 13032 produced a XADH surplus of $369 \%$ which decreased to $346 \%$ in the pgi deletion mutant. An increased surplus of $538 \%$ was determined for C. glutamicum $\triangle p u r A$ $\triangle g u a B 2$. This difference can be ascribed to the low activity of the PPP (29\%) and the contrariwise high TCA cycle activity (88\%). In comparison to C. glutamicum $\triangle$ purA $\triangle$ guaB2, C. glutamicum $\triangle$ purA $\triangle$ guaB2 purF ${ }^{K 348 Q} \Delta$ pgi exhibited a decreased XADH surplus of $458.2 \%$.

The consequences of the unequal NADH/FADH $\mathrm{H}_{2}$ supply are discussed in more detail in "Cluster analysis" (see section Discussion). 


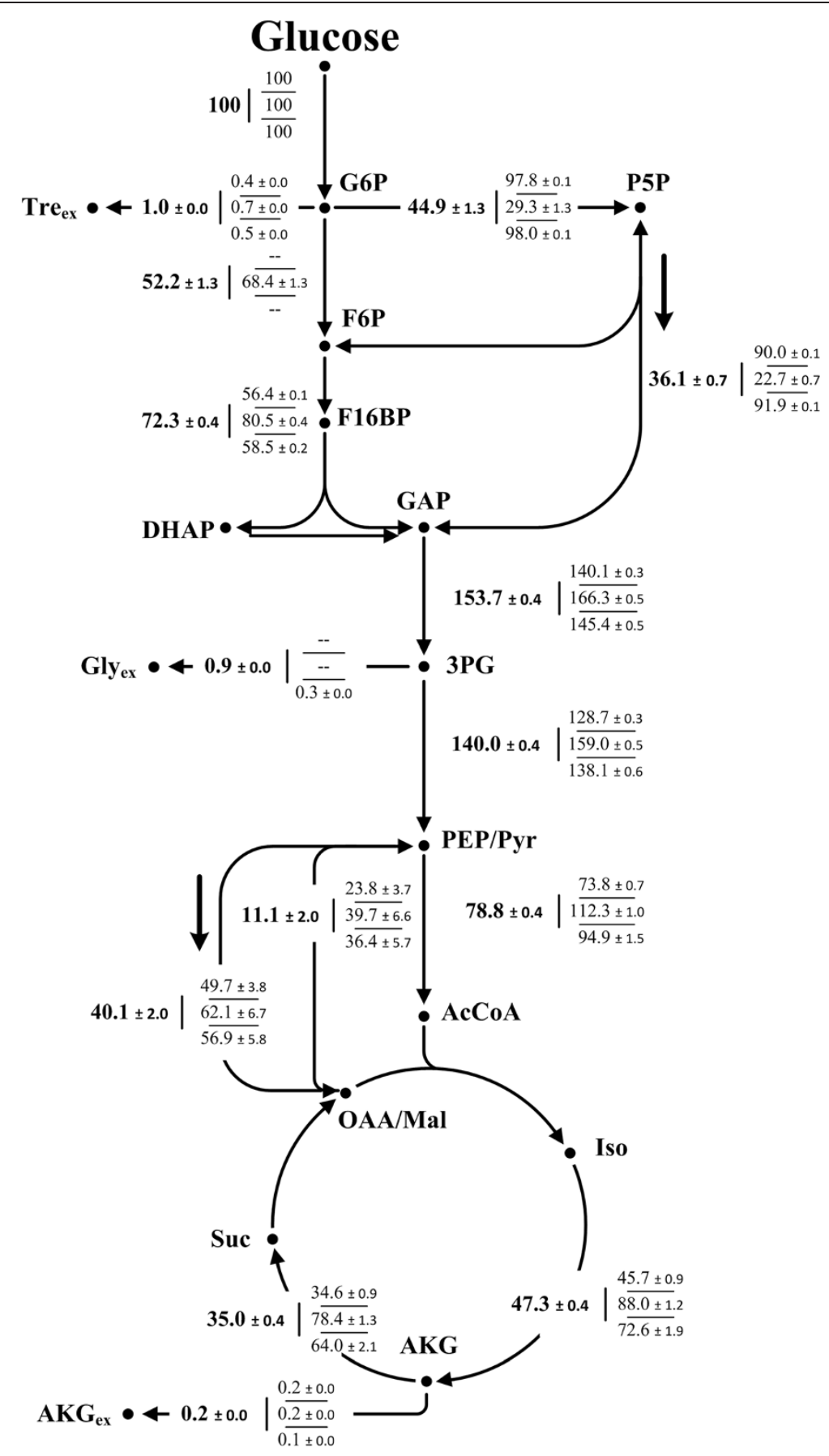

Figure 2 Intracellular carbon flux distributions with corresponding standard deviations of the central metabolism of the wild type C. glutamicum ATCC 13032 (in bold) and mutant strains C. glutamicum $\triangle$ pgi, C. glutamicum $\triangle$ purA $\triangle$ guaB2 and C. glutamicum $\triangle$ purA $\triangle$ guaB2 purF ${ }^{K 48 Q} \Delta p g i$ (displayed in that order from top to bottom). Net fluxes are given as molar percentage of the mean specific glucose uptake rates (4.67 mmol g-1 $\mathrm{h}^{-1}$ for C. glutamicum ATCC 13032; $4.18 \mathrm{mmol} \mathrm{g}^{-1} \mathrm{~h}^{-1}$ for C. glutamicum $\Delta p g ; ; 4.24 \mathrm{mmol} \mathrm{g}^{-1} \mathrm{~h}^{-1}$ for C. glutamicum $\triangle$ purA $\triangle$ guaB2; $1.92 \mathrm{mmol} \mathrm{g}^{-1} \mathrm{~h}^{-1}$ for C. glutamicum $\triangle$ purA $\triangle$ guaB2 purF ${ }^{K 3480} \Delta$ pgi), which were set to $100 \%$. Anabolic fluxes leading towards biomass formation were not displayed for the benefit of lucidity. Anabolic demands for auxtrophic mutant strains C. glutamicum $\triangle p u r A \triangle$ guaB2 and C. glutamicum $\triangle$ purA $\triangle$ guaB2 pur ${ }^{K 3480} \Delta$ pgi were corrected for the supplementation of adenine and guanine. Abbreviations: G6P, glucose 6-phosphate, P5P, pentose 5-phosphate; F6P, fructose 6-phosphate; F16BP, fructose 1,6-bisphosphate; DHAP, dihydroxyacetone phosphate; GAP, glyceraldehyde 3-phosphate; 3PG, 3-phosphoglycerate; PEP, phosphoenolpyruvate; Pyr, pyruvate; AcCoA, acetyl-CoA; Iso, isocitrate; AKG, a-ketoglutarate; Suc, succinate; $\mathbf{O A A}$, oxaloacetate; Mal, malate; $\mathbf{G l y}_{\mathbf{e x}}$, extracellular glycine; Tre $_{\mathbf{e x}}$, extracellular trehalose. 


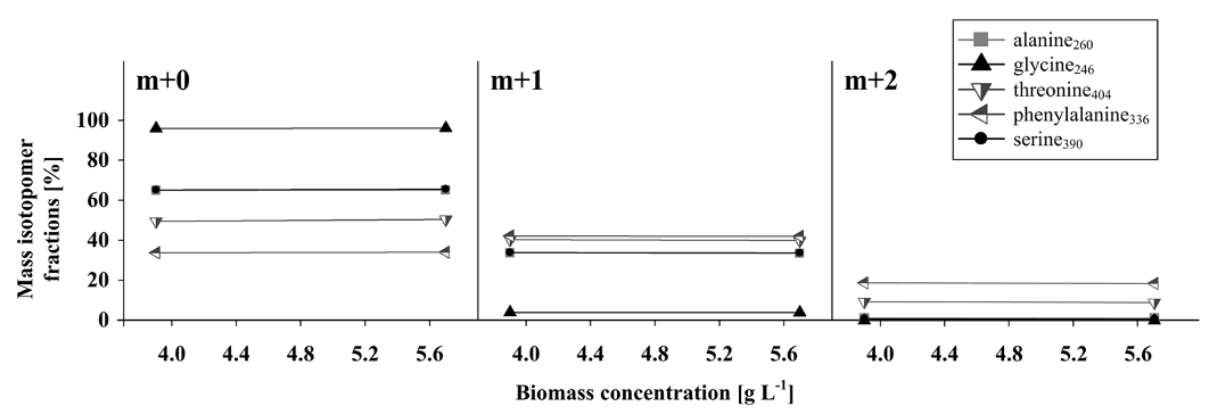

Figure 3 Mass isotope fractions [\%] of amino acid [M-57] fragments derived from different precursor molecules throughout the central carbon metabolism. Data are shown exemplarily for C. glutamicum ATCC 13032.

\section{Discussion}

Rerouting metabolic fluxes - contributions of individual pathway branches with respect to purine accumulation Targeted perturbations in the purine biosynthetic pathway Imbalances in free intracellular purine pools are reported to be mutagenic and genotoxic [20,21]. Thus a prerequisite for balanced cellular growth is to maintain the intracellular pool sizes, which resulted in efficient regulatory mechanisms $[1,16,17,33,35,38]$. This pool stability is on the one hand sustained by an interaction of de novo purine synthesis and the salvage pathway, ensuring a constant supply of purine nucleotides required for cellular growth [16]. On the other hand, increasing concentrations perturbing the system can easily be broken down via the present degrading and converting reactions.

In case of the purA gene deletion mutant (C. glutamicum $\triangle p u r A$ ), extracellular supplementation with adenine completely restored cellular growth, resulting in comparable specific growth rates of the wild type $\left(0.443 \mathrm{~h}^{-1}\right)$ and the

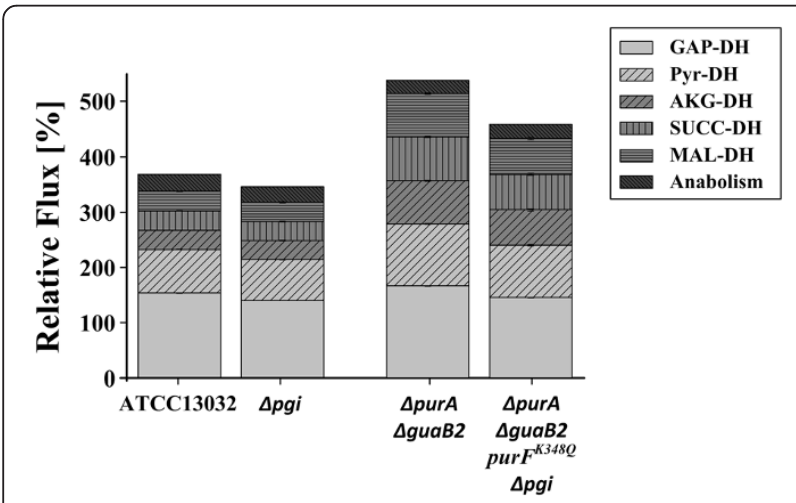

Figure $4 \mathrm{XADH}$ formation for C. glutamicum ATCC 13032, C. glutamicum $\triangle p g i, C$. glutamicum $\triangle p u r A \triangle$ guaB2 and C. glutamicum $\triangle$ purA $\triangle$ guaB2 purF $^{K 348 Q} \Delta$ pgi. Glyceraldehyde 3-phosphate dehydrogenase (GAP-DH), pyruvate dehydrogenase (Pyr-DH), a-ketoglutarate dehydrogenase (AKG-DH) and malate dehydrogenase (MAL-DH) were considered as NADH supplying reactions and succinate dehydrogenase (SUCC-DH) as FADH ${ }_{2}$ supplying reaction. Anabolically derived NADH was considered with $3.2 \mathrm{mmol} \mathrm{g}_{\mathrm{CDW}}^{-1}[40]$.
C. glutamicum $\triangle$ purA strain $\left(0.455 \mathrm{~h}^{-1}\right)$, indicating a thorough compensatory effect by the salvage pathway.

On the contrary, extracellular guanine complementation was not sufficient to restore wild type growth behavior in the guaB2 gene deletion mutant (C. glutamicum $\triangle$ guaB2): specific growth rate as well as biomass yield were reduced by $25 \%$, each compared to the wild type strain (Table 2 ). This can either be explained by insufficient uptake or converting systems for guanine. The latter is catalyzed by the salvage pathway. The observed phenotypic consequences reemerged in every genotype comprising this genetic perturbation (C. glutamicum $\triangle g u a B 2$ and $C$. glutamicum $\triangle$ purA $\triangle$ guaB2), no matter if single or multiple genes were deleted. The attribution of this fundamental salvage branch restriction regarding uptake or conversion could not be answered definitely in this study. Decreasing intracellular guanine concentrations in C. glutamicum $\triangle$ guaB2, however indicate an uptake insufficiency (and not a conversion insufficiency!). Decreasing metabolite concentrations are caused by increased consuming reactions (in this case by the salvage pathway).

In contrast, the purA gene deletion mutant C. glutamicum $\triangle p u r A$ exhibited increasing adenine concentrations, supporting a sufficient adenine uptake, even exceeding converting reactions by the salvage pathway. This theory is additionally supported by the reduced guanine uptake (125 $\left.\mu \mathrm{mol} \mathrm{g}_{\mathrm{CDW}}^{-1}\right)$ compared to the adenine uptake (243 $\left.\mu \mathrm{mol} \mathrm{g}_{\mathrm{CDW}}^{-1}\right)$, indicating basic differences for both intermediates.

Regarding intracellular pool sizes, the single gene deletion mutants C. glutamicum $\triangle$ purA and C. glutamicum $\triangle g u a B 2$ were found to exhibit the most promising rises in intracellular IMP concentration with maximal IMP pool sizes of 44.0 and $37.2 \mu \mathrm{mol} \mathrm{g}_{\mathrm{CDW}}^{-1}$, respectively. These associated transient concentration changes showed no relation to biomass formation and resulted in unstable and thus unrepresentative results. This phenomenon may at least partially be associated with direct changes in the GMP and AMP pool sizes (Table 1). These two compounds are reported to act as the most 
stringent regulators for the purine biosynthetic pathway by a feedback-mediated inhibition of the PurF enzyme [16]. With both intermediates operating as regulators, it is not surprising that the individual gene deletions cause spreading influences on the whole branching pathway.

\section{Targeted perturbation in the central carbon metabolism}

Increasing the precursor supply via the oxidative branch of the PPP seemed to be a promising route to boost intracellular purine levels. This strategy has already been successfully applied for tryptophan production in E. coli [39] and lysine production in C. glutamicum [40,41], illustrating positive correlations between enhanced productivity and increased PPP activities [42].

Thus, the genetic modification (deletion of the pgi gene) was implemented into the genome. The resulting affects on the purine intermediate pool sizes (Table 1) however, were rather limited compared to those observed for purA and guaB2 gene deletion strains, indicating the strong regulation and rigidity of this pathway.

\section{Effects of combined perturbations on intracellular purine pool sizes}

Strains used for industrial manufacturing of purine intermediates are usually characterized by an adenine auxotrophy $[35,37,43-45]$. This perturbation seems to be fundamental, revealing the importance of an IMP conversion block for the accumulation of this intermediate metabolite [46]. The extracellular supplementation with adenine, however, is linked to a repression of transcription initiation [37] of the genes of the pur operon [37] via the depletion of PRPP as described for B. subtilis. In order to counterbalance this phenomenon, the carbon flux was rerouted by introducing the $p g i$ gene deletion, aiming at an increased PRPP supply (Figure 2).

The carbon flux was successfully rerouted. Intracellular purine levels though further shifted from nucleotides towards their degradation products, mainly inosine and hypoxanthine (Table 1).

\section{Cluster analysis}

In order to analyze correlations between the different manipulated pathway branches, principal component analysis (PCA) was performed. The result of the cluster analysis is illustrated in Figure 5. Pareto-scaled concentrations of purine pathway intermediates were used for the PCA, resulting in the global purine metabolite phenotype for each mutant.

The first two components contributed to more than $90 \%$ of the total variance. As shown in the score plot, a distinct separation pattern defining two groups could be derived (Figure 5). The first group (A.), comprising the wild type strain, the pgi deletion strain and the purF substituent, did not show any significant differences concerning intracellular purine intermediate pool sizes. As discussed earlier, only minor changes with respect to intracellular concentration of purine pathway intermediates were caused by these genetic manipulations, which in direct comparison to the second group (B.) were quite consistent. The second group (B.), however, resulted in a broader clustering pattern comprising intrinsic dissimilarities. As shown by loading coefficients (Figure 6), the separation was mainly caused by the component IMP and its degradation product hypoxanthine, reflecting the observed shift from nucleotide accumulation towards increased degradation.

The disruption of critical nodes in the purine pathway itself, i.e. the conversion of IMP to AMP and GMP, provided the required fundamental basis for far-reaching effects when combined with additional modifications as the pgi gene deletion.

Solely based on the metabolome data, no clear link between the additional genetic manipulation of the pgi deletion and the increased degradation shift could be concluded. Therefore, the research was extended by an in-depth fluxome analysis to capture integrative effects of fluxome and metabolome.

\section{Connectivity of targeted metabolome and fluxome analysis}

In the context of purine accumulation, alterations in the PPP-TCA cycle ratio were of special interest, as their relative activities and especially the flux partitioning into these two main branches, mainly dictate the XADH $\left(\mathrm{NADH}+\mathrm{FADH}_{2}\right)$ supply and thus ATP formation in the respiratory chain [47]. The exclusive metabolization of glucose 6-phosphate via the PPP caused a major carbon flux redistribution in $C$. glutamicum $\triangle p u r A \triangle g u a B 2$ $\operatorname{purF}^{K 348 Q} \Delta p g i$, exhibiting an adverse effect on XADH formation compared to C. glutamicum $\triangle$ purA $\triangle$ guaB2 (Figure 4) and initiating a shift in the metabolic profile towards inosine and especially hypoxanthine formation. Since most XADH is generated in the TCA cycle, high PPP activities inevitable lead to an increased $\mathrm{CO}_{2}$ production via a decarboxylation reaction in the oxidative part of the PPP and thus to a reduced carbon availability for other metabolic branches, as seen for C. glutamicum $\triangle p u r A \Delta g u a B 2$ purF ${ }^{K 348 Q} \Delta p g i$ (Figure 2). Within the scope of the tightly regulated purine biosynthesis, Noguchi et al. [47] presumed a connection between a declining ATP supply and an increasing degrading efficiency within this pathway. The results obtained in this study are consistent with those obtained previously [47].

This suggests further metabolic engineering targets to increase intracellular IMP levels focusing on IMP degrading reactions. 

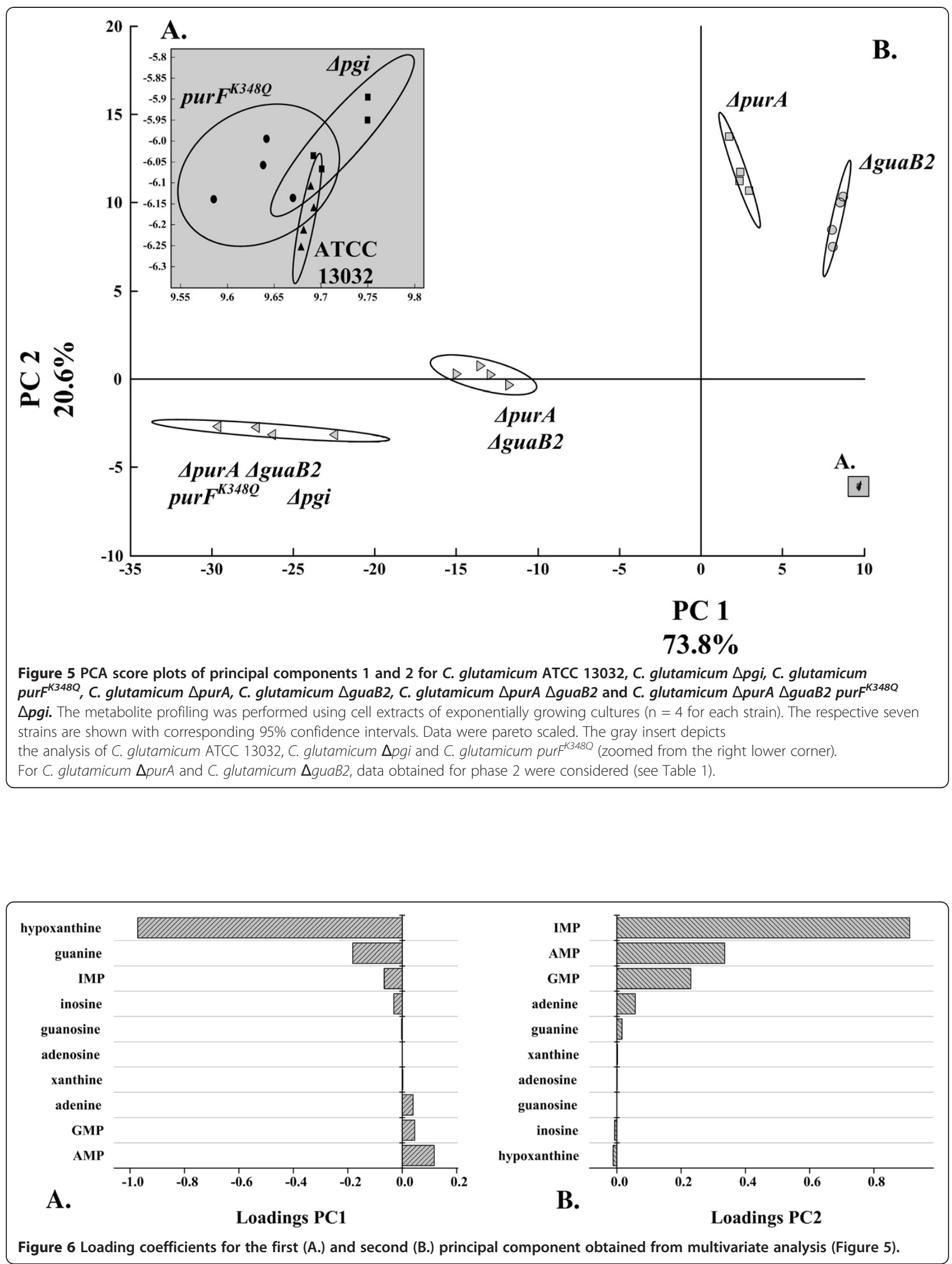


\section{Conclusions}

In the present study, we performed an in-depth metabolic profiling of the purine biosynthetic pathway in $C$. glutamicum. Site-directed perturbations on the genetic level with subsequent studies of growth behavior and targeted metabolome analysis revealed a tight regulation of the underlying pathway with strong additional effects on the carbon flux distributions in the main metabolism as determined for C. glutamicum $\triangle p u r A \triangle g u a B 2$ and C. glutamicum $\triangle p u r A \triangle g u a B 2$ purF $^{K 348 Q} \Delta p g i$.

Our results underline the importance of combined analyses employing different hierarchical levels such as fluxome and metabolome to reveal trends which indicate active regulation mechanism. The regulatory processes maintaining balanced intracellular concentrations have not been fully discovered yet and necessitate further investigation. Complex future studies will thus focus on the redox and energy metabolism, which were beyond the scope of the present analyses. In addition, it might be interesting to further analyze the metabolic instabilities - detected for C. glutamicum $\triangle p u r A$ and C. glutamicum $\triangle g u a B 2$. These instabilities were caused by the alteration of the complex regulatory structure of this pathway branch, opening up a way for in-depth surveys of this central and essential pathway in prokaryotes as well as in eukaryotes.

In order to achieve an understanding of the global cellular response, individual parts of the underlying network, i.e. precursor and cofactor supply, energy and redox metabolism, should not be assumed as independent, individual but interconnected units, linked by various key metabolites such as ATP or NAD(P)H.

\section{Methods}

\section{Organisms and plasmids}

Wild type C. glutamicum ATCC 13032 was obtained from the American Type Culture Collection (Manassas, VA, USA) and used as parent strain for further genetic modifications. E. coli $\mathrm{DH} 5 \alpha$ was utilized as host strain for cloning and plasmid construction. E. coli NM522 ${ }^{\mathrm{pTC}}$ served as host for plasmid amplification and coryne-specific methylation. The pTC plasmid, harboring an origin of replication for E. coli (ori), a tetracycline resistance $\left(\mathrm{Tet}^{\mathrm{R}}\right)$ as selection marker and the DNA-methyltransferase gene from $C$. glutamicum, was co-expressed in E. coli NM522 $2^{\mathrm{pTC}}$ to transfer the methylation pattern of C. glutamicum to the integrative plasmids. The vector pClik was used for integrative genetic modifications. The modifications constructed in this study comprised single gene deletions ( $\triangle p u r A, \triangle g u a B 2$ and $\Delta p g i$ ), an allelic replacement of the purF gene encoding amidophosphoribosyltranferase with a purFK348Q gene (purF ${ }^{K 348 Q}$ ) and multiple gene deletions ( $\triangle p u r A \triangle g u a B$ and $\triangle p u r A \Delta g u a B 2$ purF $\left.^{K 348 Q} \Delta p g i\right)$. The site-directed mutants and corresponding genetic modifications are summarized in Table 3. Allelic replacement of the modified purF gene was carried out by first deleting the native gene followed by a replacement step with the manipulated gene containing the point mutation. Primer sequences used for construction and verification are listed in Table 4.

\section{DNA preparation and recombinant DNA modifications}

Oligonucleotide synthesis was performed by SigmaAldrich (Steinheim, Germany) and DNA sequencing was carried out by GATC (Konstanz, Germany). Plasmid construction and DNA purification were performed using standard techniques. Preparation of genomic DNA from C. glutamicum was carried out with the MasterPure ${ }^{\mathrm{TM}}$ Gram Positive DNA Purification Kit (Biozym Scientific $\mathrm{GmbH}$, Germany). PCR reactions were performed in a TGradient-Cycler (Whatman-Biometra ${ }^{\mathrm{R}}$ ) with Phusion polymerase (Biozym) for insert construction and JumpStart REDTaq Ready Mix (Sigma-Aldrich) for strain verification. PCR products were purified using NucleoSpin ${ }^{\circledR}$ Extract II Kit (Macherey-Nagel, Düren, Germany) and plasmids using HiYield ${ }^{\circledR}$ Plasmid Mini Kit (SLG, Gauting, Germany). Ligations were performed with Fast-Link ${ }^{\mathrm{TM}}$ DNA Ligation Kit (Epicentre Biotechnologies, Madison, WI, USA). FastDigest ${ }^{\text {B }}$ restriction enzymes were obtained from Fermentas (St. Leon-Rot, Germany) as well as 10 mM dNTP Mix and 1-kb DNA O'GeneRuler ${ }^{\mathrm{TM}}$.

\section{Transformation}

Precultures used for transformation of C. glutamicum, were grown on $\mathrm{BHI}^{++}$medium $(10 \mathrm{~mL}$ in $100 \mathrm{~mL}$ baffled shake flasks) and incubated for $12 \mathrm{~h}$ at $30^{\circ} \mathrm{C}$. The main culture in $\mathrm{BHI}^{++}$medium $(80 \mathrm{~mL}$ in $1000 \mathrm{~mL}$ baffled shake flasks) was inoculated from exponentially growing precultures to an optical density $\mathrm{OD}_{660 \mathrm{~nm}}$ of $0.2 .400 \mathrm{mg}$ isonicotinic acid hydrazide, $2.5 \mathrm{~g}$ glycine and $0.1 \mathrm{~mL}$ Tween 80 were dissolved in $20 \mathrm{~mL} \mathrm{H}_{2} \mathrm{O}$, sterilized by filtration and added to the main culture at an $\mathrm{OD}_{660 \mathrm{~nm}}$ of 0.6. Cells were further incubated until an $\mathrm{OD}_{660 \mathrm{~nm}}$ of 0.8 was reached and harvested by centrifugation $(7 \mathrm{~min}$, $4000 \mathrm{~g}, 4^{\circ} \mathrm{C}$, Laborfuge 400R, Heraeus, Hanau, Germany). Cells were washed three times with precooled wash buffer (10\% glycerine, $8 \mathrm{mM}$ tris hydrochloride, $\mathrm{pH} 7.4$ ) and once with $10 \%$ glycerine. After the second resuspension in $6 \mathrm{~mL} \mathrm{10 \%} \mathrm{glycerine,} 200 \mu \mathrm{L}$ cells were subsequently used for transformation. Electroporation was performed using a BioRad Gene Pulser II (Hercules, California, USA) with the following parameters: $25 \mu \mathrm{F}, 600 \Omega$ and $2.5 \mathrm{kV}$. Cells were transferred into a $2 \mathrm{~mL}$ Eppendorf tube, mixed with $1 \mathrm{~mL}$ BHIS medium, followed by a heat shock for $5 \mathrm{~min}$ at $46^{\circ} \mathrm{C}$. Regeneration was performed for $90 \mathrm{~min}$ at $30^{\circ} \mathrm{C}$ to allow recovery of the cells, which were then spread on BHISKan agar plates and incubated for 4 days. 
Table 3 Genetically modified organisms constructed in the present study: Mutant strains of C. glutamicum based on the wild type strain (ATCC 13032) were constructed by site-directed mutagenesis

\begin{tabular}{|c|c|c|}
\hline Strain & Genotype & Reference \\
\hline C. glutamicum ATCC 13032 & wild type & $\begin{array}{l}\text { American Type } \\
\text { Culture Collection }\end{array}$ \\
\hline C. glutamicum $\Delta p g i$ & deletion of the pgi gene ( $\mathrm{NCgl0817)}$ & This work \\
\hline C. glutamicum $\triangle p$ pur & deletion of the purA gene ( $\mathrm{NCg} / 2669)$ & This work \\
\hline C. glutamicum $\triangle$ guaB2 & deletion of the guaB2 gene ( $\mathrm{NCgl0578)}$ & This work \\
\hline C. glutamicum pur ${ }^{K 348 Q}$ & Nucleotide exchange $\mathrm{K} 348 \mathrm{Q}$ in the purF gene (NCgl2495) & This work \\
\hline C. glutamicum $\triangle$ purA $\triangle$ guaB2 & double deletion of purA and guaB2 genes & This work \\
\hline $\begin{array}{l}\text { C. glutamicum } \triangle \text { purA } \triangle \text { guaB2 } \\
\text { purf }^{348} \Delta p g i\end{array}$ & $\begin{array}{l}\text { deletion of purA, guaB2 and pgi genes and allelic amino acid } \\
\text { substitution } \mathrm{K} 348 \mathrm{Q} \text { in the purF gene }\end{array}$ & This work \\
\hline E. coli DH5a & 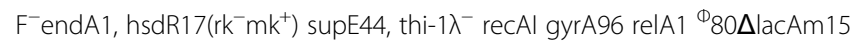 & [48] \\
\hline E. coli NM522 $2^{\mathrm{pTC}}$ & 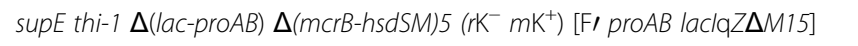 & Stratagene \\
\hline
\end{tabular}

E. coli strains were employed for plasmid construction.

Preparation of heat shock competent E. coli cells was performed as described by Inoue et al. [49]. Transformation of $E$. coli $\mathrm{DH} 5 \alpha$ and $\mathrm{NM} 522^{\mathrm{pTC}}$ with the integrative pClik-derivates was performed by heat shock [49]. Briefly, cells were incubated on ice for $30 \mathrm{~min}$, heated at $45^{\circ} \mathrm{C}$ for $45 \mathrm{~s}$ and again cooled on ice for $2 \mathrm{~min}$. Regeneration was performed for $1 \mathrm{~h}$ at $37^{\circ} \mathrm{C}$ after $900 \mu \mathrm{L}$ SOC medium were added.

\section{Targeted mutant generation}

Site-directed mutations were introduced using the integrative vector pClik, which lacks an ori for C. glutamicum. Selection on $\mathrm{Kan}^{\mathrm{R}}$ as resistance marker yielded transformed cells with genome-integrated plasmid DNA. The insertion of the plasmids thereby results from a single crossover recombinant event. The second homologous recombination event, generated by the $s a c B$ positive selection system [50], causes the deletion of the $s a c B$ gene and therefore allows growth on corresponding selective agar plates. Due to the $s a c B$ associated additional deletion of the native or the modified gene sequence, transformants either contain the wild type gene sequence or exhibit the targeted mutation. Kan ${ }^{\mathrm{R}}$ nonresistant and sucrose-insensitive clones were further verified by allelic-specific PCR for the presence of the targeted gene sequence. The corresponding gene sequence for a nucleotide exchange, i.e. $\operatorname{purF}^{K 348 Q}$, was first deleted and then replaced.

\section{Chemicals}

Yeats extract, beef extract, polypeptone, casamino acids, and brain heart infusion (BHI) were purchased from Difco Laboratories (Detroit, USA). 99\% enriched glucose, i.e. $\left[1-{ }^{13} \mathrm{C}\right]$ and $\left[1,2-{ }^{13} \mathrm{C}_{2}\right]$, were obtained from

Table 4 Mutant genotypes and corresponding site-specific primer sequences ( $F$, forward; $R$, reverse) used for verification $^{\text {a }}$

\begin{tabular}{|c|c|c|}
\hline Mutant genotype & Primer & Primer sequence \\
\hline \multirow[t]{2}{*}{$\overline{\Delta p g i}$} & $\Delta p g i-\mathrm{F}$ & 5/-GATCACGCGTATCCCTTCTCCGGCATC-3I \\
\hline & $\Delta p g i-\mathrm{R}$ & 5/-GATCTCTAGATCCAGCGACACGAATAATC-3' \\
\hline \multirow[t]{2}{*}{$\Delta p u r A$} & $\Delta p u r A-F$ & 5/-GATCTCTAGAATGGATCGGATGTGACG-3/ \\
\hline & $\Delta p u r A-\mathrm{R}$ & 5/-GATCACGCGTCAATCGGTCAACCTGGT-3/ \\
\hline \multirow[t]{2}{*}{$\triangle g u a B 2$} & $\Delta g u a B 2-F$ & 5/-GATCACGCGTGAGTITCTACCGGAGGA-31 \\
\hline & $\Delta g u a B 2-R$ & 5/-GATCTCTAGATCAGACTGGAAGTAACG-3" \\
\hline \multirow[t]{4}{*}{ purF ${ }^{K 348 Q}$} & purF $^{K 348 Q_{-} F}$ & 5/-GATCACGCGTGATTGCGGACTGGTTAC-31 \\
\hline & purF $F^{K 348 Q_{-R}}$ & 5/-GATCTCTAGACTCCTGCTGCTGCGTATG-3/ \\
\hline & K348Q-1 & 5/-GGCCAAGGCATGGTCCAGAACGCCTACGTTGGC-3/ \\
\hline & K348Q-2 & 5/-GCCAACGTAGGCGTTCTGGACCATGCCTTGGCC-31 \\
\hline
\end{tabular}

\footnotetext{
${ }^{a}$ For the single amino acid substitution in purF ${ }^{K 348 Q}$, sequences for fusion primers (K348Q-1, K348Q-2) containing the point mutation (underlined) are denoted.
} 
Cambridge Isotope Laboratories (Andover, MA., USA). All other chemicals and reagents of analytical grade were purchased from Sigma-Aldrich, Merck (Darmstadt, Germany), Fluka (Buchs, Switzerland) or Roth (Karlsruhe, Germany).

\section{Growth Media}

The first precultures were grown on complex media $(\mathrm{pH}$ 6.8) containing per liter: $10.0 \mathrm{~g}$ glucose, $2.5 \mathrm{~g} \mathrm{NaCl}, 2.0 \mathrm{~g}$ urea, $5.0 \mathrm{~g}$ yeast extract, $5.0 \mathrm{~g}$ beef extract, $5.0 \mathrm{~g}$ polypeptone and $20.0 \mathrm{~g}$ casamino acids. For agar plates, the complex medium was supplemented with $20.0 \mathrm{~g} \mathrm{~L}^{-1}$ agar. Second precultures and main cultures were grown on minimal medium ( $\mathrm{pH} 7.2)$ containing per liter: $15.0 \mathrm{~g}$ glucose, $4.0 \mathrm{~g} \mathrm{KH}_{2} \mathrm{PO}_{4}, 16.0 \mathrm{~g} \mathrm{Na}_{2} \mathrm{HPO}_{4}, 500 \mathrm{mg} \mathrm{MgCl} \mathrm{mg}_{2}$. $6 \mathrm{H}_{2} \mathrm{O}, 300 \mathrm{mg}$ 3,4-dihydroxibenzoic acid, $100 \mathrm{mg} \mathrm{CaCl} \mathrm{C}_{2}$. $2 \mathrm{H}_{2} \mathrm{O}, 100 \mu \mathrm{g}$ cyanocobalamin, $750 \mu \mathrm{g}$ thiamine, $4 \mu \mathrm{g}$ pyridoxal phosphate, $100 \mu \mathrm{g}$ biotin, $400 \mu \mathrm{g}$ calcium pantothenate, $2 \mu \mathrm{g}$ folic acid, $400 \mu \mathrm{g}$ nicotinic acid, 200 $\mu \mathrm{g}$ 4-aminobenzoic acid, $400 \mu \mathrm{g}$ pyridoxine $\cdot \mathrm{HCl}, 2 \mathrm{mg}$ inositol, $10 \mathrm{mg} \mathrm{FeCl} \cdot 4 \mathrm{H}_{2} \mathrm{O}, 1 \mathrm{mg} \mathrm{ZnCl}, 100 \mu \mathrm{g} \mathrm{CuCl}_{2}$, $20 \mu \mathrm{g} \mathrm{NiCl} 2 \cdot 6 \mathrm{H}_{2} \mathrm{O}, 20 \mu \mathrm{g} \mathrm{Na}_{2} \mathrm{MoO}_{4} \cdot 2 \mathrm{H}_{2} \mathrm{O}, 500 \mu \mathrm{g}$ boric acid, $100 \mu \mathrm{g} \mathrm{KI}, 100 \mu \mathrm{g} \mathrm{CoCl}_{2} \cdot 6 \mathrm{H}_{2} \mathrm{O}$ and $10 \mathrm{mg}$ $\mathrm{MnCl}_{2} \cdot 4 \mathrm{H}_{2} \mathrm{O}$. For tracer studies, naturally labeled glucose was replaced by $99 \%{ }^{13} \mathrm{C}$ enriched $\left[1-{ }^{13} \mathrm{C}\right]$ or $\left[1,2-{ }^{13} \mathrm{C}\right]$ glucose. Mutants of $C$. glutamicum exhibiting deletions of adenylosuccinate synthetase (purA) or IMP dehydrogenase (guaB2) were grown on minimal media additionally supplemented with $200 \mathrm{mg} \mathrm{L}^{-1}$ adenine sulfate or $80 \mathrm{mg} \mathrm{L}^{-1}$ guanine hydrochloride, due to the introduced auxotrophies.

Media used for transformation experiments of C. glutamicum contained per liter: $37 \mathrm{~g}$ BHI, 26.4 g $\left(\mathrm{NH}_{4}\right)$ ${ }_{2} \mathrm{SO}_{4}$ and $40 \mathrm{~g}$ glucose for $\mathrm{BHI}^{++}$and $37 \mathrm{~g} \mathrm{BHI}$ and $46 \mathrm{~g}$ sorbitol for BHIS medium.

\section{Cultivations}

Cultivations were carried out in baffled shake flasks on an orbital shaker (Multitron 2, Infors AG, Bottmingen, Switzerland) at $230 \mathrm{rpm}$ and $30^{\circ} \mathrm{C}$. First precultures were grown on complex medium $(25 \mathrm{~mL}$ medium in $250 \mathrm{~mL}$ baffled flask) for $8 \mathrm{~h}$ and used for inoculation of the second precultures $(25 \mathrm{~mL}$ medium in $250 \mathrm{~mL}$ baffled flask). Second precultures and main cultures were performed in minimal medium. Main cultivations were performed in duplicates in minimal medium using $500 \mathrm{~mL}$ baffled shake flasks with $50 \mathrm{~mL}$ medium. For tracer experiments, which were conducted under the same conditions as main cultivations, naturally labeled glucose was replaced by $\left[1-{ }^{13} \mathrm{C}\right]$ glucose or $\left[1,2-{ }^{13} \mathrm{C}_{2}\right]$ glucose for analysis of glucose 6-phosphate isomerase (pgi) deletion mutants and the volume was adjusted to $5 \mathrm{~mL}$ in $50 \mathrm{~mL}$ baffled shake flasks.

\section{Substrate and product analysis}

Biomass concentration was determined by measuring the optical density of the culture at $660 \mathrm{~nm}\left(\mathrm{OD}_{660 \mathrm{~nm}}\right)$ using a photometer (Marsha Pharmacia Biotech, Freiburg, Germany) which was gravimetrically correlated with the cell dry weight The correlation factor was determined as $0.414 \mathrm{~g}_{\mathrm{CDW}} \mathrm{OD}_{660 \mathrm{~nm}}^{-1}$. Extracellular substrates and secreted products were analyzed after $3 \mathrm{~min}$ centrifugation at $16,000 \mathrm{~g}$. Quantification of glucose, $\alpha$-ketoglutarate and trehalose was performed in diluted supernatants by HPLC (Kontron Instruments, Neufahrn, Germany). Separation was performed using an Aminex HPX-87H Bio-Rad column (300 x $7.8 \mathrm{~mm}$; Hercules, USA), at $55^{\circ} \mathrm{C}$ with a constant flow rate of $0.8 \mathrm{~mL} \mathrm{~min}^{-1}\left(7 \mathrm{mM} \mathrm{H}_{2} \mathrm{SO}_{4}\right.$ as mobile phase). Refraction index (RI) detection was used for quantification of sugars, and UV detection at $218 \mathrm{~nm}$ for organic acids and at $254 \mathrm{~nm}$ for quantification of supplemented adenine and guanine. Determination of amino acids was performed as described previously [36]. Extracellular concentrations of adenine and guanine were determined by HPLC (Agilent 1290 Infinity; Agilent Technologies, Waldbronn, Germany) using a Phenomenex (Aschaffenburg, Germany) reversed-phase C18-column (Gemini $5 \mathrm{u} 110 \AA$, 150 x $4.6 \mathrm{~mm}$ ). Separations were carried out at $10^{\circ} \mathrm{C}$ with a constant flow of $0.8 \mathrm{~mL} \mathrm{~min}{ }^{-1}$. Gradient elution was performed using $5 \mathrm{mM}$ ammonium formate solution at $\mathrm{pH} 5.8$ (eluent A) and acetonitrile: methanol:water mixture (45:45:10, eluent B) applying the following gradient: an initial isocratic step with 0\% B (3.5 min) was followed by an increase to $30 \%$ within $10 \mathrm{~min}$ and to $100 \%$ within $1 \mathrm{~min}$. The flushing step at $100 \% \mathrm{~B}$ was performed for $5 \mathrm{~min}$. The column was reconditioned at $0 \% \mathrm{~B}$ for another $5 \mathrm{~min}$.

\section{Analysis of intracellular metabolites}

Extraction and quantification of intracellular purine intermediates, i.e. nucleotides, nucleosides and nucleobases, by LC-ESI-MS/MS was performed as described previously [28].

\section{Mass spectrometric ${ }^{13} \mathrm{C}$-labeling analysis}

The mass isotopomer distributions of amino acids derived from cellular protein and of trehalose secreted during cultivation were determined by mass spectrometry-gas chromatography. Sample preparation and instrument settings were performed as described previously [41,51,52]. In order to ensure isotopic steady state, labeling patterns of amino acids and trehalose were determined for two biomass concentrations during mid-exponential growth. Metabolic (pseudo-) steady state was assessed by constant yields, uptake and secretion rates and constant intracellular pool sizes of purine intermediates. 


\section{Fluxome analysis}

Calculation of the in vivo metabolic fluxes of the C. glutamicum strains was carried out by fitting simulated mass isotopomer distributions to experimentally determined labeling patterns of amino acids derived from cellular protein and trehalose secreted during cultivation. The calculations were based on the approach of Yang et al. [53] implemented in Matlab (version 2008b, Mathworks Inc., Nattick, USA) using metabolite and isotopomer balancing. Anabolic demands for biomass synthesis and extracellular production rates ware additionally considered [54] and implemented in the metabolic network. Media supplementation with adenine and guanine required by strains comprising adenylosuccinate synthetase and/or IMP dehydrogenase deletions - led to reduced anabolic demands for DNA and RNA syntheses. The corresponding reduced anabolic demand for 3phosphoglycerate was considered. In addition, increased ribose 5-phosphate requirements due to increased intracellular pool sizes of IMP, determined for C. glutamicum $\triangle$ purA $\triangle$ guaB2 purF $^{K 348 Q} \triangle p g i$ and C. glutamicum $\triangle$ purA $\triangle$ guaB2, were considered likewise.

Measured GC-MS data were corrected for naturally occurring isotopes in the corresponding amino acid fragments and derivatization reagent respectively [55,56]. A Monte Carlo approach with 100 independent runs was employed for the statistical analysis [57].

\section{Data analysis}

Mean concentration values for purine intermediates were calculated from four biological replicates for each strain for two biomass concentrations and corrected using ${ }^{13} \mathrm{C}$-labeled cell extract as internal standard as described previously [28]. The metabolomics-based phenotype profiling was extended by principal component analysis (PCA) applying pareto scaling of the data. Data sets were compressed by reduction of dimension numbers and the metabolomic patterns were highlighted. PCA was performed using Matlab R2007b (The Math Works, Inc., Boston, USA) software.

\section{Competing interests}

The authors declare that they have no competing interests.

\begin{abstract}
Authors' contributions
$\mathrm{SP}, \mathrm{KS}, \mathrm{EH}$ were involved in conception and design of experiments. SP, TB, $\mathrm{SZ}$ performed the targeted strain engineering and cultivation experiments. SP carried out analytical quantitation supported by DAV and KS. SP, KS, DAV and $\mathrm{EH}$ were involved in manuscript preparation. KS and SP were involved in data analysis and interpretation. KS drafted and revised the manuscript. All authors read and approved the final manuscript.
\end{abstract}

\section{Acknowledgments}

DAV acknowledges research support by the Alfried Krupp von Bohlen und Halbach-Stiftung.

\section{Author details}

'Biochemical Engineering Institute, Saarland University, Campus A1.5, 66123, Saarbrücken, Germany. ${ }^{2}$ Institute of Bioanalytical Chemistry, Saarland University, Campus B2.2, 66123, Saarbrücken, Germany.

Received: 31 August 2012 Accepted: 21 October 2012 Published: 24 October 2012

\section{References}

1. Pang B, McFaline $J$, Burgis NE, Dong M, Taghizadeh K, Sullivan MR, Elmquist CE, Cunningham RP, Dedon PC: Defects in purine nucleotide metabolism lead to substantial incorporation of xanthine and hypoxanthine into DNA and RNA. Proc Natl Acad Sci USA 2012, 109:2319-2324.

2. Traut TW: Physiological concentrations of purines and pyrimidines. $\mathrm{Mol}$ Cell Biochem 1994, 140:1-22.

3. Cordell RL, Hill SJ, Ortori CA, Barrett DA: Quantitative profiling of nucleotides and related phosphate-containing metabolites in cultured mammalian cells by liquid chromatography tandem electrospray mass spectrometry. J Chromatogr B Analyt Technol Biomed Life Sci 2008, 871:115-124.

4. Guimaraes PM, Londesborough J: The adenylate energy charge and specific fermentation rate of brewer's yeasts fermenting high- and very high-gravity worts. Yeast 2008, 25:47-58.

5. Halpern BP: What's in a name? Are MSG and umami the same? Chem Senses 2002, 27:845-846.

6. Shimaoka M, Kawasaki H, Takenaka Y, Kurahashi O, Matsui H: Effects of edd and pgi disruptions on inosine accumulation in Escherichia coli. Biosci Biotechnol Biochem 2005, 69:1248-1255.

7. Matsui H, Shimaoka M, Takenaka Y, Kawasaki H, Kurahashi O: gsk disruption leads to guanosine accumulation in Escherichia coli. Biosci Biotechnol Biochem 2001, 65:1230-1235.

8. Cohen S, Jordheim LP, Megherbi M, Dumontet C, Guitton J: Liquid chromatographic methods for the determination of endogenous nucleotides and nucleotide analogs used in cancer therapy: a review. J Chromatogr B 2010, 878:1912-1928.

9. Hofmann U, Heinkele G, Angelberger S, Schaeffeler E, Lichtenberger C, Jaeger S, Reinisch W, Schwab M: Simultaneous quantification of eleven thiopurine nucleotides by liquid chromatography-tandem mass spectrometry. Anal Chem 2012, 84:1294-1301.

10. Li H, Zhang G, Deng A, Chen N, Wen T: De novo engineering and metabolic flux analysis of inosine biosynthesis in Bacillus subtilis. Biotechnol Lett 2011, 33:1575-1580.

11. Abbouni B, Elhariry HM, Auling G: Overproduction of $\mathrm{NAD}^{+}$and 5 '-inosine monophosphate in the presence of $10 \mu \mathrm{M} \mathrm{Mn}^{2+}$ by a mutant of Corynebacterium ammoniagenes with thermosensitive nucleotide reduction $\left(n r d^{\text {ts }}\right)$ after temperature shift. Arch Microbiol 2004, 182:119-125.

12. Furuya $A$, Abe $S$, Kinoshita S: Production of nucleic acid-related substances by fermentation processes XXXIII. Accumulation of inosine by a mutant of Brevibacterium ammoniagenes. Appl Microbiol 1970, 20:263-270

13. Furuya A, Abe S, Kinoshita S: Production of nucleic acid-related substances by fermentative processes. XIX. Accumulation of 5'-inosinic acid by a mutant of Brevibacterium ammoniagenes. Appl Microbio/ 1968, 16:981-987.

14. Bebenek K, Roberts JD, Kunkel TA: The effects of dNTP pool imbalances on frameshift fidelity during DNA replication. J Biol Chem 1992, 267:3589-3596.

15. Tozzi MG, Camici M, Mascia L, Sgarrella F, Ipata PL: Pentose phosphates in nucleoside interconversion and catabolism. FEBS J 2006, 273:1089-1101.

16. Messenger $L$, Zalkin H: Glutamine phosphoribosylpyrophosphate amidotransferase from Escherichia coli Purification and properties. J Biol Chem 1979, 254:3382-3392.

17. Saxild HH, Nygaard P: Regulation of levels of purine biosynthetic enzymes in Bacillus subtilis: effects of changing purine nucleotide pools. J Gen Microbiol 1991, 137:2387-2394.

18. Abbouni B, Elhariry HM, Auling G: Arrest of cell cycle by inhibition of ribonucleotide reductase induces accumulation of $\mathrm{NAD}^{+}$by $\mathrm{Mn}^{2+}$-supplemented growth of Corynebacterium ammoniagenes. Biotechnol Lett 2003, 25:143-147. 
19. Stephanopoulos G, Vallino JJ: Network rigidity and metabolic engineering in metabolite overproduction. Science 1991, 252:1675-1681.

20. Mathews CK: DNA precursor metabolism and genomic stability. FASEB J 2006, 20:1300-1314.

21. Wheeler L, Rajagopal I, Mathews CK: Stimulation of mutagenesis by proportional deoxyribonucleoside triphosphate accumulation in Escherichia coli. DNA Repair (Amst) 2005, 4:1450-1456.

22. Nielsen J: It is all about metabolic fluxes. J Bacterio/ 2003, 185:7031-7035.

23. Fiehn $\mathrm{O}$ : Metabolomics-the link between genotypes and phenotypes. Plant Mol Biol 2002, 48:155-171.

24. van Gulik WM, Heijnen JJ: A metabolic network stoichiometry analysis of microbial growth and product formation. Biotechnol Bioeng 1995, 48:681-698.

25. Fiehn $\mathrm{O}$ : Combining genomics, metabolome analysis, and biochemical modelling to understand metabolic networks. Comp Funct Genomics 2001, 2:155-168.

26. Nielsen J, Oliver S: The next wave in metabolome analysis. Trends Biotechnol 2005, 23:544-546.

27. Dunn WB: Current trends and future requirements for the mass spectrometric investigation of microbial, mammalian and plant metabolomes. Phys Biol 2008, 5:011001.

28. Peifer S, Schneider K, Nürenberg G, Volmer DA, Heinzle E: Quantitation of intracellular purine intermediates in different Corynebacteria using electrospray LC-MS/MS. Anal Bioanal Chem 2012. doi:10.1007/s00216-012-6388-6.

29. Provost A, Bastin G: Dynamic metabolic modelling under the balanced growth condition. J Process Control 2004, 14:717-728.

30. Wittmann C: Fluxome analysis using GC-MS. Microb Cell Fact 2007, 6:6.

31. Marx A, de Graaf A, Wiechert W, Eggeling L, Sahm H: Determination of the fluxes in the central metabolism of Corynebacterium glutamicum by Nuclear Magnetic Resonance Spectroscopy combined with metabolite balancing. Biotechnol Bioeng 1996, 49(2):111-129.

32. Wiechert W: ${ }^{13} \mathrm{C}$ metabolic flux analysis. Metab Eng 2001, 3:195-206.

33. Shimaoka M, Takenaka Y, Kurahashi O, Kawasaki H, Matsui H: Effect of amplification of desensitized purF and prs on inosine accumulation in Escherichia coli. J Biosci Bioeng 2007, 103:255-261.

34. Zhou G, Charbonneau H, Colman RF, Zalkin H: Identification of sites for feedback regulation of glutamine 5-phosphoribosylpyrophosphate amidotransferase by nucleotides and relationship to residues important for catalysis. J Biol Chem 1993, 268:10471-10481.

35. Matsui H, Kawasaki H, Shimaoka M, Kurahashi O: Investigation of various genotype characteristics for inosine accumulation in Escherichia coli W3110. Biosci Biotechnol Biochem 2001, 65:570-578.

36. Kromer JO, Fritz M, Heinzle E, Wittmann C: In vivo quantification of intracellular amino acids and intermediates of the methionine pathway in Corynebacterium glutamicum. Anal Biochem 2005, 340:171-173.

37. Qian J, Cai X, Chu J, Zhuang Y, Zhang S: Nucleotide mutations in purA gene and pur operon promoter discovered in guanosine- and inosine-producing Bacillus subtilis strains. Biotechnol Lett 2006, 28:937-941.

38. Zhou G, Smith JL, Zalkin H: Binding of purine nucleotides to two regulatory sites results in synergistic feedback inhibition of glutamine 5-phosphoribosylpyrophosphate amidotransferase. J Biol Chem 1994, 269:6784-6789.

39. Mascarenhas D, Ashworth DJ, Chen CS: Deletion of pgi alters tryptophan biosynthesis in a genetically engineered strain of Escherichia coli. Appl Environ Microbiol 1991, 57:2995-2999.

40. Becker J, Klopprogge C, Herold A, Zelder O, Bolten CJ, Wittmann C: Metabolic flux engineering of L-lysine production in Corynebacterium glutamicum-over expression and modification of G6P dehydrogenase. J Biotechnol 2007, 132:99-109.

41. Becker J, Klopprogge C, Zelder O, Heinzle E, Wittmann C: Amplified expression of fructose 1,6-bisphosphatase in Corynebacterium glutamicum increases in vivo flux through the pentose phosphate pathway and lysine production on different carbon sources. Appl Environ Microbiol 2005, 71:8587-8596.

42. Wittmann C, Becker J: The L-lysine story: From metabolic pathways to industrial production. Springer Berlin/ Heidelberg: Microbiology Monographs; 2007.

43. Mori H, lida A, Fujio T, Teshiba S: A novel process of inosine 5'-monophosphate production using overexpressed guanosine/inosine kinase. Appl Microbiol Biotechnol 1997, 48:693-698.
44. Kamada N, Yasuhara A, Takano Y, Nakano T, Ikeda M: Effect of transketolase modifications on carbon flow to the purine-nucleotide pathway in Corynebacterium ammoniagenes. Appl Microbiol Biotechnol 2001, 56:710-717.

45. Demain AL, Jackson M, Vitali RA, Hendlin D, Jacob TA: Production of guanosine-5'-monophosphate and inosine-5'-monophosphate by fermentation. Appl Microbiol 1966, 14:821-825.

46. Villas-Boas SG, Hojer-Pedersen J, Akesson M, Smedsgaard J, Nielsen J: Global metabolite analysis of yeast: evaluation of sample preparation methods. Yeast 2005, 22:1155-1169.

47. Noguchi $Y$, Shimba N, Kawahara Y, Suzuki E, Sugimoto S: ${ }^{31}$ P NMR studies of energy metabolism in xanthosine-5'-monophosphate overproducing Corynebacterium ammoniagenes. Eur J Biochem 2003, 270:2622-2626.

48. Hanahan D: Studies on transformation of Escherichia coli with plasmids. J Mol Biol 1983, 166:557-580.

49. Inoue $H$, Nojima $H$, Okayama $H$ : High efficiency transformation of Escherichia coli with plasmids. Gene 1990, 96:23-28.

50. Jager W, Schafer A, Puhler A, Labes G, Wohlleben W: Expression of the Bacillus subtilis $s a c B$ gene leads to sucrose sensitivity in the gram-positive bacterium Corynebacterium glutamicum but not in Streptomyces lividans. J Bacteriol 1992, 174:5462-5465.

51. Kiefer P, Heinzle E, Zelder O, Wittmann C: Comparative metabolic flux analysis of lysine-producing Corynebacterium glutamicum cultured on glucose or fructose. Appl Environ Microbiol 2004, 70:229-239.

52. Wittmann C, Kiefer P, Zelder O: Metabolic fluxes in Corynebacterium glutamicum during lysine production with sucrose as carbon source. Appl Environ Microbiol 2004, 70:7277-7287.

53. Yang $\mathrm{TH}$, Frick $\mathrm{O}$, Heinzle E: Hybrid optimization for ${ }^{13} \mathrm{C}$ metabolic flux analysis using systems parametrized by compactification. BMC Syst Biol 2008, 2:29.

54. Wittmann C, Heinzle E: Metabolic network analysis and design in Corynebacterium glutamicum. In In Corynebacteria - Genomics and Molecular Biology. Edited by Burkovski A. Norfolk UK: Caister Academic Press; 2008:79-112.

55. van Winden WA, Wittmann C, Heinzle E, Heijnen JJ: Correcting mass isotopomer distributions for naturally occurring isotopes. Biotechnol Bioeng 2002, 80:477-479.

56. Wittmann C, Heinzle E: Mass spectrometry for metabolic flux analysis. Biotechnol Bioeng 1999, 62:739-750.

57. Wittmann C, Heinzle E: Genealogy profiling through strain improvement by using metabolic network analysis: metabolic flux genealogy of several generations of lysine-producing corynebacteria. Appl Environ Microbiol 2002, 68:5843-5859.

doi:10.1186/1475-2859-11-138

Cite this article as: Peifer et al:: Metabolic engineering of the purine biosynthetic pathway in Corynebacterium glutamicum results in increased intracellular pool sizes of IMP and hypoxanthine. Microbial Cell Factories 2012 11:138.

\section{Submit your next manuscript to BioMed Central and take full advantage of:}

- Convenient online submission

- Thorough peer review

- No space constraints or color figure charges

- Immediate publication on acceptance

- Inclusion in PubMed, CAS, Scopus and Google Scholar

- Research which is freely available for redistribution 